\title{
Morbillivirus de los Delfines: Patógeno re-emergente en la población de cetáceos
}

\author{
Manuela Echeverri-Zuluaga ${ }^{1,}$ Yeison H. Duque-García ${ }^{1}, J u l i a n$ Ruiz-Saenz ${ }^{2 \bowtie}$
}

\section{Dolphin Morbillivirus: Re-emerging pathogen in cetacean population}

\begin{abstract}
Dolphin morbillivirus (DMV) is one of the most important pathogen of cetaceans. It was first described in the late 80 s, since has been causing outbreaks that affects dolphin populations and also in other cetaceans in the U.S. and in the Mediterranean sea. We conducted a bibliographic search in MedLine, SciELO, Scopus ${ }^{\circledR}$ and Google Scholar without timeline limits using MeSH terms such as Cetacean morbillivirus, Pilot Whale morbillivirus, Dolphin morbillivirus, Strandings, etc., with the aim of provide a critical update on basic, clinical and epidemiological aspects of one of the most important emerging infections for cetacean wildlife, with particular emphasis on the current alternatives for diagnosis and control. Based on the information gathered we concluded that there is not only a need to study and quantify the strandings, but we need to develop awareness of the importance of this viral infection in cetaceans and to apply effective management plans that maintain those populations in biological balance.
\end{abstract}

Keywords: cetacean morbillivirus; epidemiology; Paramyxoviridae; pilot whale Morbillivirus; RT-PCR.

Edited by Alberto Acosta $\square$

1. Grupo de Investigación CENTAURO, Facultad de Ciencias Agrarias, Universidad de Antioquia, Medellín, Colombia.

2 Grupo de Investigación en Ciencias Animales - GRICA, Facultad de Medicina Veterinaria y Zootecnia, Universidad Cooperativa de

Colombia, sede Bucaramanga.

Received: 26-04-2014 Accepted: 12-05-2014

Published on line: 10-07-2014

Citation: Echeverri-Zuluaga M, Duque-García YH, Ruiz-Saenz J (2015) Morbillivirus de los Delfines: Patógeno re-emergente en la población de cetáceos. Universitas Scientiarum 20(1): 29-41

doi: 10.11144/Javeriana.SC20-1.mdpr

Funding: Programa de Sostenibilidad 2013-2014 del Comité para el Desarrollo de la Investigación - CODI de la Universidad de Antioquia.

Electronic supplementary material: N/A

\section{Introducción}

Desde finales de los años $80 \mathrm{y}$ hasta la actualidad, el Morbillivirus cetáceo ha emergido como el patógeno de mayor importancia en este grupo de animales causando grandes epidemias en los océanos Atlántico, Pacífico y Mediterráneo principalmente (Fowler \& Cubas 2001).

Hasta mediados de 1988 se pensó que el género Morbillivirus, perteneciente a la familia Paramixoviridae, estaba conformado por cuatro tipos de virus: el virus del sarampión (MV), el virus del moquillo canino (CDV), el virus de la peste bovina y el virus de la peste de los pequeños rumiantes; y es a partir de 1994 en que se añade a este género una nueva especie: el morbillivirus cetáceo ó "Cetacean morbillivirus" (CeMV) el cual se ha 
subdividido en tres grupos claramente diferenciados: el morbillivirus de las marsopas (PMV; Kennedy 1998), el morbillivirus de los delfines (DMV; Osterhaus et al. 1992, Barrett et al. 1993) y el morbillivirus de las ballenas piloto (PWMV; Taubenberger et al. 2000).

Recientemente, se ha visto un gran aumento de muertes o varamientos de delfines asociadas a la infección por el CeMV; por lo que la presente revisión presenta una actualización crítica sobre los aspectos básicos, clínicos y epidemiológicos de una de las infecciones emergentes más importantes para la fauna cetácea mundial, haciendo especial énfasis en las alternativas actuales para su diagnóstico y control. Para lograr este propósito se siguió una estrategia heurística de búsqueda bajo el uso de palabras clave de interés (Morbillivirus, Cetacean, Dolphin, etc.) en idioma inglés para ampliar la captura de información y sus combinaciones mediante el uso de conectores boléanos y funciones de límites en las bases de datos MedLine, SciELO, Scopus ${ }^{\circledR}$ y Google Scholar.

Caracterización de los CeMV: Los CeMV son virus de genoma RNA de una sola hebra de sentido negativo; puntualmente el DMV está compuesto de 15.702 bases (NCBI Ref Seq NC_005283.1; Figura 1) que contiene seis genes los cuales codifican para ocho proteínas, dos no estructurales y seis estructurales que se organizan en sentido 3 '- 5 ': nucleoproteína $(\mathrm{N})$ fosfoproteína $(\mathrm{P})$ - proteína de la matriz $(\mathrm{M})$ - proteína de fusión $(\mathrm{F})$ - proteína hemaglutinina $(\mathrm{H})$ - y $(\mathrm{L})$ proteína grande (Rima et al. 2005).

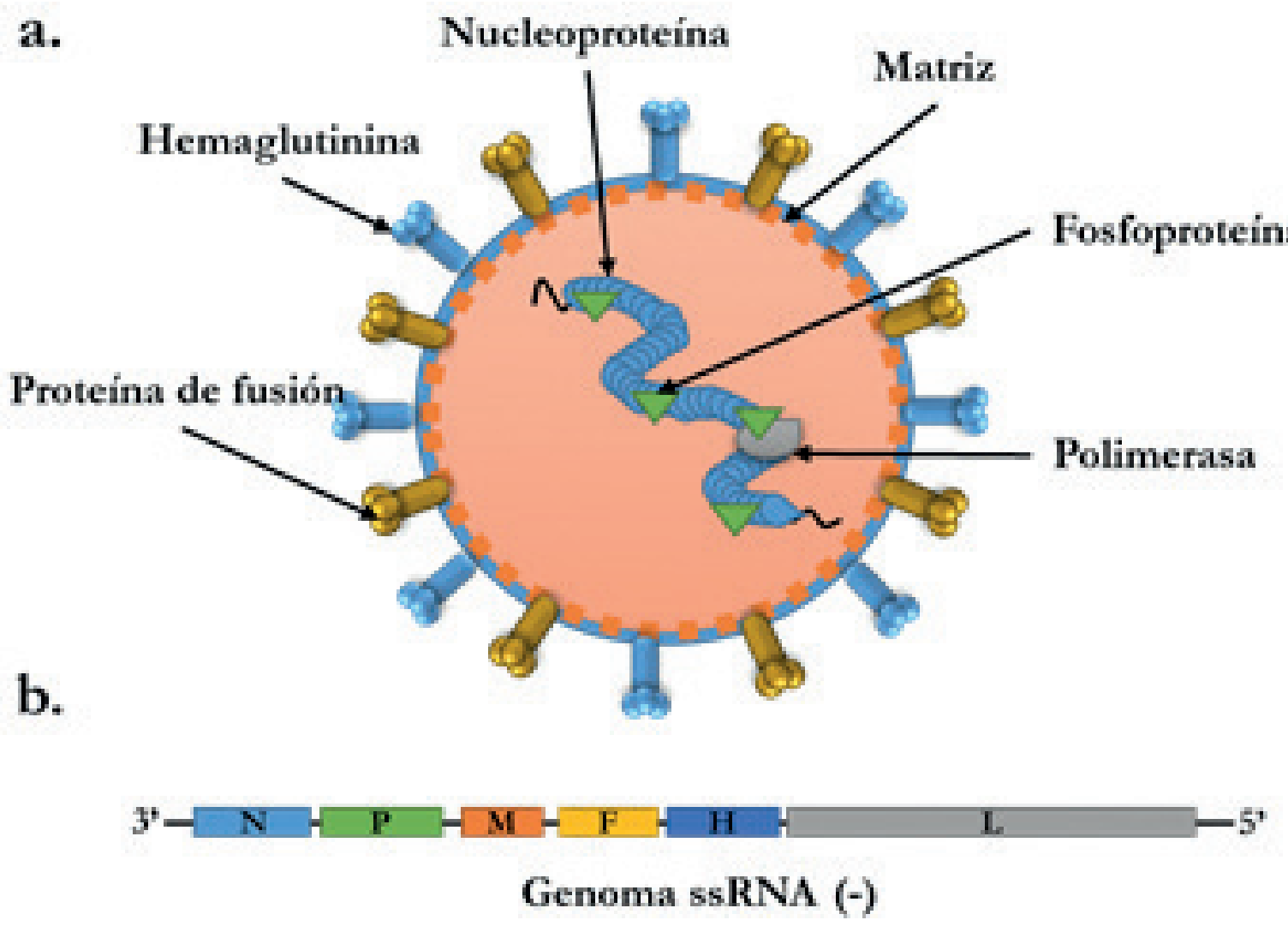

Fig. 1. Morfología del virón del CeMV y organización del genoma. a) Diagrama esquemático de una partícula del CeMV en un corte transversal: P, fosfoproteína; L, Polimerasa; M, matriz o proteína de membrana; F, proteína de fusión; N, nucleoproteína; H, hemaglutinina. En negro el RNA viral. b). Mapa del RNA genómico (3 '- 5') del CeMV. Cada caja representa un mRNA codificado por separado. 
ElDMV fue descrito por primera vez en 1990, en un varamiento de delfines listados (Stenella coeruleoalba) en la costa mediterránea de España; en dicho varamiento, las muertes comenzaron en la costa de Valencia y se extendieron a las costas mediterráneas francesas y del norte de África. Los principales hallazgos patológicos encontrados indicaron la presencia de encefalitis y neumonía intersticial difusa. En estos animales se encontró la presencia de antígenos de Morbillivirus en los pulmones, el cerebro, los ganglios linfáticos, y el epitelio biliar y fue posible aislar un Morbillivirus de algunos tejidos (Domingo et al. 1990).

El PMV se describió por primera vez en marsopas comunes (Phocoena phocoena) durante un brote de moquillo en la costa de Irlanda del Norte, en las cuales se detectó la presencia de antígeno de morbillivirus en inclusiones intracitoplasmáticas en neuronas $\mathrm{y}$ astrocitos al igual que en el epitelio bronquial y bronquiolar (Kennedy 1998).

Recientemente, estudios realizados en una ballena piloto de aleta larga (Globicephalus melas) varada en costas de New Jersey (USA), demostraron

\section{a. Gen P}

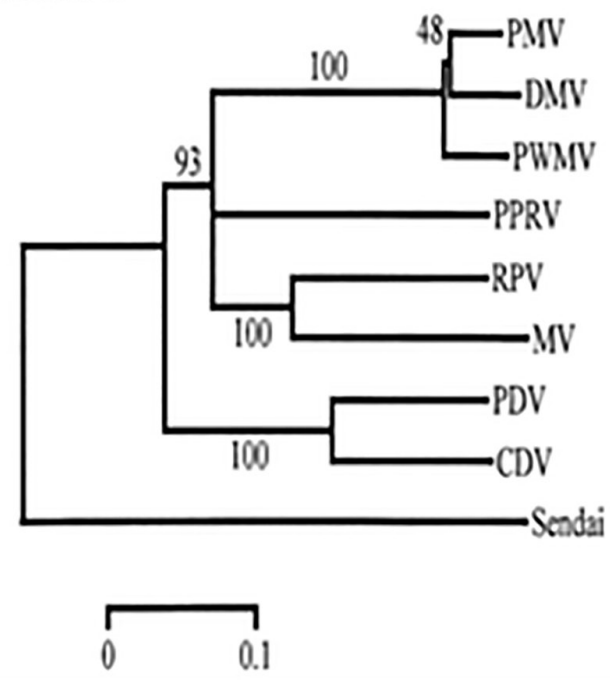

la presencia de lesiones macro y microscópicas indicativas de infección por un Morbillivirus (cuerpos de inclusión intracitoplasmáticos en diversos órganos, encefalomielitis no supurativa, etc.) y la presencia de secuencias genómicas de una nueva cepa de Morbillivirus relacionado con los CeMV, el cual fue denominado PWMV (Taubenberger et al. 2000).

En 2005, siguiendo los criterios recomendados por la Organización Mundial de la Salud para la caracterización genotípica de aislamientos del MV, los cuales se basan en amplificación y secuenciación de la región C-terminal de la proteína $\mathrm{N}$ y la secuencia completa de la hemaglutinina viral, se demostró que, debido a la baja divergencia aminoacídica entre estos, el DMV y el PMV se consideran dos cepas de una misma especie viral (Van de Bildt et al. 2005).

Filogenéticamente, es posible evidenciar la relación existente entre distintos morbillivirus de la familia Paramyxoviridae (Figura 2). Adicionalmente se puede notar como en el análisis de los genes $\mathrm{N}$ y $\mathrm{P}$, el PMWV se agrupa en la misma clada con los PMV y DMV, con un valor de soporte de rama de moderado

\section{b. Gen N}

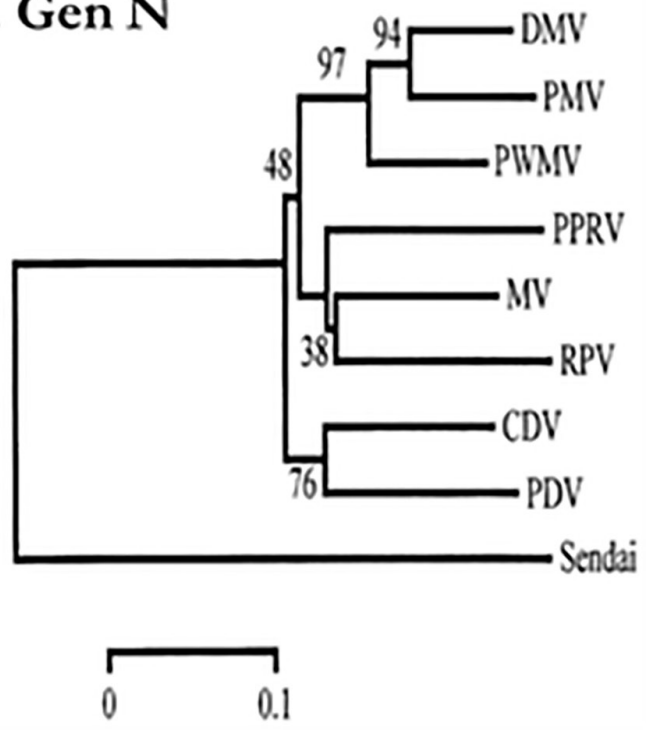

Fig. 2. Análisis filogenético de secuencias parciales de los genes $\mathrm{P}$ y $\mathrm{N}$ usando el método Neighbor-joining indicando distancias de rama. a) Para el gen P se muestra el análisis de una secuencia de 378 nucleótidos. b) Para el gen N se analizó un fragmento de 203 nucleótidos. PMV: morbillivirus de las marsopas; DMV: morbillivirus de los delfines; PWMV: morbillivirus de las ballenas piloto; PPRV: virus de la peste de pequeños rumiantes; RPV: Virus de la peste Bovina; MV: Virus del Sarampion; PDV: Virus del Distemper de focas; CDV: Virus del Distemper canino; Sendai: Virus de parainfluenza murina. Adaptado de Taubenberger et al. 2000. 
a alto y claramente distante de otros morbillivirus de rumiantes y de carnívoros (Figura 2), lo cual sugiere que el PWMV posee un ancestro común con el DMV o el PMV (Taubenberger et al. 2000).

Patogénesis de la infección: El DMV es uno de los virus más patogénicos de los CeMV, afecta a diferentes especies de cetáceos, entre los cuales, los delfines mulares (Tursiops truncatus) y los delfines listados son las especies que presentan mayores tasas de mortalidad a causa del agente. Al igual que los demás miembros del género Morbillivirus, el DMV es un virus linfotrópico, epiteliotrópico y neurotrópico, por tanto y como consecuencia de la infección, los animales presentan depleción de ganglios linfáticos, lesiones pulmonares, lesiones en piel y serias alteraciones en el comportamiento que pueden ser la causa de los varamientos. Adicionalmente, se han reportado casos de delfines listados infectados, que presentan encefalitis no supurativa en grados de subaguda a crónica, con lesiones cerebrales que se asemejan mucho a las que se encuentra en humanos en la "panencefalitis esclerosante subaguda" (Domingo et al. 1995) y en caninos en las "encefalitis del perro viejo" (Soto et al. 2011). Cabe resaltar que dichas lesiones se han asociado al MV y CDV en humanos y caninos respectivamente.

Una interesante discusión se ha abierto en los últimos años acerca del mecanismo a través del cual el DMV (y otros morbillivirus) logra cruzar la barrera hemato-encefálica previa a la invasión del parénquima cerebral del huésped junto a sus poblaciones neuronales residentes. Una posible respuesta parece residir en la existencia de cepas virales con comportamiento neurotrópico selectivo y exclusivo, el cual debe estar determinado por la interacción específica del DMV con una molécula receptora de forma selectiva y consistentemente expresada por las neuronas (Di Guardo 2012, Di Guardo et al. 2013).

También es importante mencionar que hasta el momento se desconoce cómo puede variar la sensibilidad a la infección por Morbillivirus entre una especie y otra de delfines, lo que sí está claro es que la molécula receptora a nivel de los linfocitos, conocida como SLAM, es la molécula encargada de permitir la invasión y propagación del morbillivirus. Varios estudios han intentado clarificar cual es el papel del
SLAM y han encontrado que variaciones en el dominio $\mathrm{V}$ de ésta pueden acarrear mayor o menor afinidad por el morbillivirus, lo cual constituye un nuevo acercamiento en la determinación de la vulnerabilidad que algunas especies puedan tener por la infección con este virus (Di Guardo 2012). Sin embargo, un estudio publicado (Bellière et al. 2009), con base en análisis filogenéticos de secuencias de los distintos tipos de CeMV y su asociación con las tasas de mortalidad de los varamientos, la especie hospedadora y la distribución geográfica, indica que existe un grupo de CeMV (al cual pertenece el PWMV) cuyos virus tienen un rango de hospedador más restringido, una distribución geográfica más reducida, así como un porcentaje de mortalidad menor en comparación con los otros dos grupos en los cuales se encuentran los (PMV y DMV). En general, el DMV posee parámetros epidemiológicos que pueden impactar seriamente a la población de cetáceos (Bellière et al. 2009; Tabla 1).

Tabla 1. Características patológicas ligadas a los diferentes grupos de CeMV. DMV: morbillivirus de los delfines; PMV: morbillivirus de las marsopas; PWMV: morbillivirus de las ballenas piloto. $(+++)$ : Alta; $(++)$ : Moderada; (+): Baja. Tomado de Bellière et al. (2009).

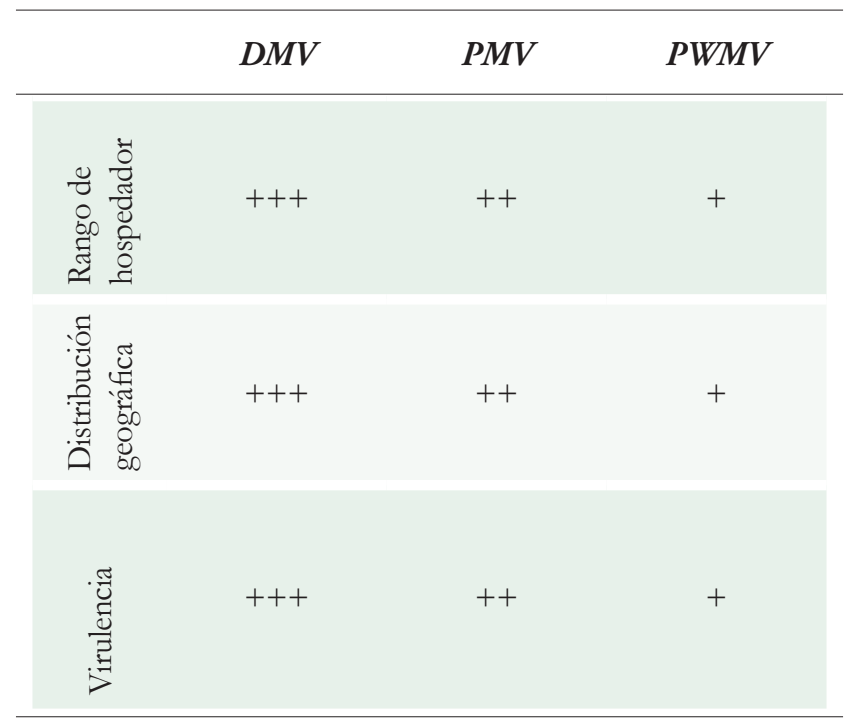

El virus se transmite probablemente por vía horizontal y por contacto directo. Hasta el momento no se ha reportado predisposición por sexo, y se ha descrito que pueden presentarse infecciones mixtas entre diferentes tipos de morbillivirus (Taubenberger et al. 1996). Adicionalmente, se han reportado 
coinfecciones entre el DMV, Brucella spp y Toxoplasma gondii las cuales pueden aumentar la patología y el daño a los animales (Van Bressem et al. 2009, Mazzariol et al. 2012). A pesar que hasta la fecha no se tienen reportes de algún potencial zoonótico del DMV (Dierauf \& Gulland 2001), los saltos de especie vistos en esta y otras especies de Morbillivirus como el CDV han generado algunas de las investigaciones que se están ejecutando actualmente, las cuales pretenden brindar nuevas perspectivas al respecto tanto para el DMV como para los demás CeMV (Ludlow et al. 2014).

Distribución y epidemiología: En América, 14 de las 18 especies de Odontocetos en el Atlántico Oeste, desde el Ártico canadiense hasta el Golfo de México, presentan anticuerpos contra el CeMV; en Centroamérica y Sudamérica, a pesar de no conocerse datos exactos respecto a la prevalencia del CeMV, existe evidencia serológica que demuestra la presencia de CeMV, particularmente en Perú (Van Bressem et al. 1998a). Lo anterior, plantea la posibilidad que también esté presente en Centroamérica, dado que varias especies cetáceas migran anualmente en dirección norte-sur a lo largo del continente americano y éstas pueden actuar como vectores y reservorios biológicos del CeMV. Esta situación supone un riesgo inminente para las demás especies de cetáceos ubicados en el centro y sur del continente americano (Fowler \& Cubas 2001). Dentro de este contexto, el estudio se centró en el impacto que el DMV ha venido teniendo y tiene sobre la población mundial de delfines.

En Norteamérica, la primera evidencia de anticuerpos contra Morbillivirus se obtuvó en delfines mulares en la costa atlántica de los Estados Unidos durante la primera epidemia ocurrida entre los años 1987 y 1988 (Geraci 1989), aunque la cifra exacta no se conoce se estima que el $50 \%$ de la población costera de estos delfines murieron durante este evento, lo que representó una mortalidad 10 veces mayor de lo normal (Scott et al. 1988, McLellan et al. 2002). Sin embargo, la mortalidad más alta registrada por el DMV, ocurrió en el período comprendido entre 1990 y 1992, en donde se registró un brote en el mar mediterráneo que afectó a los delfines listados (Aguilar \& Raga 1993). En Grecia particularmente, 63 de estos delfines murieron junto con otros 66 de cinco especies diferentes desde julio de 1991 hasta finales de diciembre 1992. La propagación del virus a lo largo de esta área continuó hacia el sur-este y luego hacia el norte-este del foco original en el mar Jónico (Daniel 1995). Años más tarde, un estudio confirmó la infección crónica por DMV en 42 delfines listados del mediterráneo, encontrando infecciones sistémicas en 36 individuos y lesiones locales cerebrales en 6 individuos (Domingo et al. 1995).

Más recientemente, entre el 2006 y el 2008, se presentaron nuevos brotes a lo largo de la costa mediterránea española y francesa, en donde tres especies diferentes de cetáceos fueron infectados por el DMV: el delfín listado, el delfín mular y la ballena piloto de aleta larga (Globicephala melas; Bellière et al. 2011). La epidemia empezó afectando a las ballenas piloto de aleta larga en el Estrecho de Gibraltar entre octubre de 2006 y abril de 2007 (Fernández et al. 2008). Específicamente, los resultados sugieren que el virus pudo realizar el salto de especie a la ballena piloto de aleta larga por la fijación de una mutación de una alanina por una valina en el dominio $\mathrm{N}$-terminal de la proteína de fusión (Bellière et al. 2011). De igual modo, se reportó la infección por DMV en una foca en cautiverio (Phoca vitulina), con hallazgos patológicos consistentes con la infección por morbillivirus. El estudio confirmó la transmisión de DMV entre especies de cetáceos y la infección en pinnípedos (Mazzariol et al. 2013). Este hallazgo es preocupante, dada la susceptibilidad documentada de las focas como la foca monje del Mediterráneo (Monachus monachus) y que están en peligro de extinción (O'Mara et al. 1999), lo cual convierte a este virus en un potencial peligro para ciertas especies, como se ha visto con el moquillo canino (CDV) que se ha convertido en un riesgo para la conservación de los tigres siberianos (Panthera tigris altaica; Seimon et al. 2013) y nuevamente resalta la importancia del salto entre especies y el posible potencial zoonótico de estos agentes virales.

En el mismo brote, en el Mediterráneo español entre Julio y Octubre de 2007, se encontraron más de 100 delfines listados varados: algunos muertos y en estado de descomposición y otros vivos con serios problemas y lesiones neurológicas que murieron tras haber sido rescatados, y a finales del 2007 a lo largo de la costa francesa del Mediterráneo, el virus afectó a más de cinco delfines mulares (Keck et al. 2010). Los resultados de los estudios realizados, mostraron que las cepas que ocasionaron las muertes de delfines y 
ballenas en este brote, son 99,9\% idénticas y deben ser consideradas el mismo DMV, además se encontró una similitud del 99,3 al 99,4\% de ese virus con la cepa española que originó el brote de 1990 (Bellière et al. 2011). Teniendo en cuenta todo lo anterior, el estudio filogenético y la zona geográfica común para los dos brotes, se sugiere que el DMV causante del brote de los años 2006-2008, evolucionó a partir de la cepa responsable del brote de 1990. Algunos años más tarde, en 2011, a lo largo de la costa Valenciana mediterránea de España, se presentó un varamiento masivo de delfines, que afectó por lo menos 26 delfines listados, tres delfines mulares y 8 delfines de especies no identificadas. Este evento, superó en sólo dos meses la tasa de mortalidad normal de 28,4 delfines/ año en esta costa (Gozalbes et al. 2010). Estudios histopatológicos y moleculares utilizando RT-PCR en tiempo real confirmaron la asociación de estos varamientos con DMV (Rubio-Guerri et al. 2013b).

Sin embargo, a pesar de los esfuerzos realizados en el estudio de la transmisión y patogénesis del DMV tanto en Europa como en América, en Julio de 2013 se presenta un aumento de la mortalidad en delfines en la costa este de los Estados Unidos, principalmente en el área comprendida entre Nueva York y Virginia. Los resultados de estudios iniciales sugieren que este aumento corresponde a un nuevo brote del DMV que está afectando a los delfines mulares. Los reportes indican que entre el 1 de Julio de 2013 y el 13 de abril de 2014 han resultado varados por lo menos 1219 delfines (Figura 3), en los cuales por lo menos 203 delfines se han confirmado como positivos para Morbillivirus (NOAA 2014).

Históricamente la mayor mortalidad reportada en la costa este de los EEUU, se alcanzó en el brote que duró desde junio de 1987 hasta mayo de 1988 en donde se tuvieron alrededor de 742 animales muertos. El comparar estos datos y determinar que este nuevo brote es uno de los más agresivos de la historia, ha llevado a que el gobierno de los Estados Unidos haya declarado un "evento de mortalidad inusual", el cual se define como: "un varamiento inesperado que implica una significativa mortandad de cualquier población de mamíferos marinos y exige una respuesta inmediata", una declaración que desde el punto de vista legal permite enviar recursos federales y científicos para ayudar a la contención inmediata del evento (NOAA 2014).

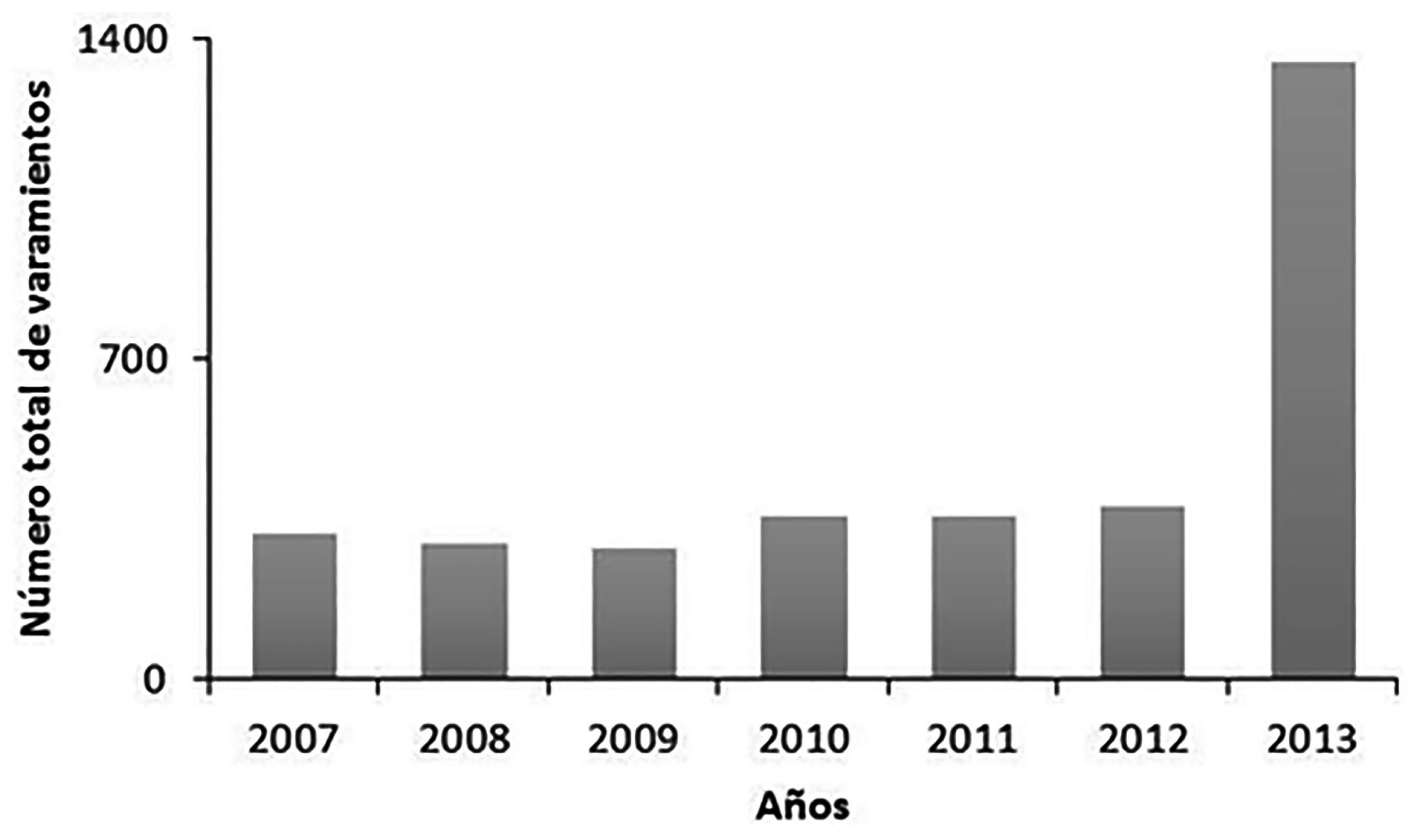

Fig. 3. Número total anual de varamientos de delfines nariz de botella por año (enero 1 a Noviembre 24) en el área geográfica comprendida entre Nueva York y la Florida (EEUU). 
Respecto a las causas reportadas de este "evento de mortalidad inusual", los resultados iniciales hacen pensar en la posibilidad que los brotes se están presentando porque los huéspedes se están haciendo más susceptibles inmunológicamente a la infección por el DMV debido a la contaminación de su ambiente. En este sentido, un estudio recientemente publicado realizó una comparación entre los dos grandes eventos epidémicos causados por el DMV (entre 1990-1992 y 2006-2008), y demostró que los niveles de organoclorados (OC) presentes en los delfines muestreados en el brote de 1990, fueron 10 veces mayores para bifenilos policlorados PCB y 6 veces mayores para DDT (Dicloro Difenil Tricloroetano), que los niveles de OC encontrados en los delfines del brote que comenzó en 2006. Sin embargo, esta investigación también concluye que, debido a que la virulencia de la primera gran epidemia fue mucho menor, y que los delfines afectados no presentaron concentraciones de OC más elevadas que los individuos aparentemente sanos. El aumento en los niveles de contaminantes organoclorados, no fue el factor primordial desencadenante del brote de 20062008 (Di Guardo et al. 2013).

Esta conclusión es controversial y debatida, ya que existen estudios que han logrado demostrar en investigaciones de campo, evidencia del efecto inmunotóxico de diferentes contaminantes ambientales en ballenas y delfines (Mori et al. 2006, 2008). Además, se ha reportado algunas propiedades inmunomoduladoras de diferentes xenobióticos persistentes en células linfoides de cetáceos tanto in vitro, como en modelos animales in vivo (Lahvis et al. 1995). Estos resultados, unidos a la opinión de varios autores (Van Bressem et al. 2009), proponen la contaminación como un importante factor de riesgo frente a la infección de DMV en cetáceos. Sin embargo, la discusión sigue abierta, en espera de nuevos estudios que demuestren claramente la relación entre estos contaminantes y la susceptibilidad de algunos cetáceos frente al DMV y otros CeMVs.

Endemia vs. Epidemia: Contrario a la fuerte presentación epidémica reportada en la actualidad para la costa este de los Estados Unidos, la comparación de los varamientos de delfines listados en el Mediterráneo occidental en los años 1990, 2007 y 2011 sugiere un cambio en la epidemiología del DMV; en 2011, la mortalidad fue aún más baja, las lesiones fueron menos graves, y sobre todo los animales jóvenes se vieron afectados (Rubio-Guerri et al. 2013a). Ya que las infecciones endémicas en la fauna se caracterizan por lesiones leves y cargas de patógenos más bajas comparadas con las infecciones epidémicas (Sinclair et al. 2006), es posible que la epidemiología del DMV en delfines listados en el Mediterráneo occidental esté cambiando de una presentación epidémica a una presentación endémica, hecho que ha sido consistentemente reportado en los últimos años (Domingo et al. 1995, Bellière et al. 2011, Soto et al. 2011) al encontrarse cronicidad en la infección en animales post-brote; lo cual resalta la urgente necesidad de desarrollar estudios serológicos en poblaciones de delfines en estado libre que permitan esclarecer el nivel de presentación y de circulación del DMV en población general, no solo en los varamientos.

Entre los diferentes estudios de los varamientos causados por DMV, se han presentado diferentes factores de riesgo que se cree pueden desencadenar la emergencia de la infección en una zona geográfica nueva o la aparición en una zona en la que se consideraba controlada (re-emergencia). El estrecho contacto estrecho entre poblaciones costeras de delfines mulares con especies estuarinas (tanto de Tursiops truncatus como de Stenella coeruleoalba u otros) en las cuales el virus sea endémico (Duignan et al. 1996) puede llevar a la aparición de un brote. La variación geográfica y la cepa asilada en los distintos brotes puede reflejar el movimiento de las especies de delfines y de los virus que las infectan; de hecho, recientemente se ha reportado que el solapamiento estacional de poblaciones costeras y estuarinas de delfines mulares en algunas épocas del año puede favorecer la propagación de la infección (Rosel et al. 2009). Igualmente se ha reportado que la densidad poblacional de delfines listados ha sido un factor determinante para el establecimiento de la infección de forma endémica en las costas mediterráneas (Forcada et al. 1994); indicando que densidad poblacional alta favorecería la aparición de una epidemia. En la re-emergencia de la infección en el Mediterráneo español en 2006-2007 (Raga et al. 2008, Van Bressem et al. 2009), se reportó que entre los individuos encontrados en los varamientos de delfines listados los individuos mayormente afectados han sido los juveniles (tanto machos como 
hembras) posiblemente debido a que los adultos habrían desarrollado inmunidad luego del anterior brote epidémico (Raga et al. 2008, Van Bressem et al. 2009); lo cual resalta la necesidad de desarrollar investigaciones en desarrollo e implementación de vacunas que permitan desarrollar inmunidad específica en dichas poblaciones.

Algunos factores de riesgo de tipo ambiental también han sido descritos como factores que pueden actuar de forma sinérgica, aumentando la severidad de la infección y favoreciendo la re-emergencia de brotes en las poblaciones de delfines listados. Entre estos factores encontramos las interacciones con la pesca, la endogamia, las migraciones, las altas cargas de contaminantes en las aguas, el aumento de las temperaturas de la zona superficial del mar y la limitada disponibilidad de presas, las cuales han mostrado tener serios impactos acumulativos sobre la dinámica poblacional del delfín listado en las costas del mediterráneo (Van Bressem et al. 2009).

Diagnóstico, prevención y control: La base para sospechar que un varamiento es causado por el DMV son las lesiones macroscópicas encontradas a la necropsia de los animales. Estas lesiones abarcan entre otros, lesiones pulmonares con ausencia de colapso total de los pulmones, múltiples focos de atelectasia y presencia ocasional de bronconeumonía exudativa en los lóbulos apicales, con agrandamiento y edema de los ganglios linfáticos asociados al pulmón. Macroscópicamente se ha reportado la presencia de signos compatibles con encefalitis necrotizante multifocal y se ha observado estomatitis ulcerativa en la lengua o en las encías de los animales varados. También es posible encontrar cambios en la coloración de la grasa subcutánea con o sin presencia de edema subcutáneo acompañados de condiciones corporales pobres o subóptimas (Domingo et al. 1992, Di Guardo et al. 2013).

Desde el punto de vista histológico las lesiones más frecuentemente reportadas son a nivel pulmonar la neumonía bronquiolo-intersticial y la presencia de células sincitiales en lesiones neumónicas. A nivel del sistema nervioso central la principal lesión reportada es la encefalitis no-supurativa difusa caracterizada principalmente por la presencia de manguitos perivasculares y presencia de sincitios en lesiones cerebrales, y a nivel del tejido linfoide la lesión principalmente reportada es la depleción y necrosis con presencia de células sincitiales en nódulos linfoides mediastínicos, mesentéricos y pre-escapulares (Domingo et al. 1992, Di Guardo et al. 2013).

En cuanto a la confirmación molecular del DMV, el cerebro, los nódulos linfoides y los pulmones son los tejidos en los cuales el antígeno y genoma del Morbillivirus pueden ser detectados en delfines y en otros cetáceos como la ballena piloto de aleta larga (Fernández et al. 2008). Para la detección del genoma, el método más utilizado es la reacción en cadena por polimerasa con transcripción reversa (RT-PCR; Barrett et al. 1993, Reidarson et al. 1998), usando cebadores para el gen de la Fosfoproteína del DMV; y aunque para análisis filogenéticos posteriores, es posible utilizar secuencias de los genes de la nucleoproteína como de la fosfoproteína, este último es el más utilizado (Taubenberger et al. 2000; Figura 2). Se han diseñado cebadores específicos para la detección del DMV mediante RT-PCR en tiempo real, los cuales también pueden ser utilizados en una RT-PCR convencional (Grant et al. 2009) con un producto de amplificación esperado de 173 nucleótidos del domino C-terminal del gen $\mathrm{N}$ de los CeMV (Tabla 2) el cual permite diferenciar DMV de PMV. Por sus características tanto para diagnóstico convencional como en PCR en tiempo real, esta es una de las pruebas más recomendadas en la actualidad (Rubio-Guerri et al. 2013b). Sin embargo, recientemente se ha reportado una nueva variación de RT-PCR en tiempo real que utiliza sondas pertenecientes a Universal Probe Library (UPL; Roche®) y cebadores específicos para CeMV que amplifican un segmento del gen que codifica para la proteína de fusión, la cual presenta mayor precisión, sensibilidad y especificidad para la detección y diferenciación de los DMV, PMV y PMWV (RubioGuerri et al. 2013b). Esta diferenciación, cobra gran importancia cuando se presentan varamientos masivos y epidemias que afectan diversas especies de cetáceos y se requiere determinar el agente causal específico involucrado. Se han descrito diferentes cebadores y sondas para realizar el diagnóstico y caracterización tanto del DMV como de otros CeMV los cuales pueden ser útiles por su rápido y sensible resultado en condiciones de campo (Tabla 2).

La presencia de anticuerpos en suero puede confirmarse mediante el uso de pruebas como el ELISA indirecto (Van Bressem et al. 1998a), el ELISA competitivo y la seroneutralización (Saliki et al. 2002). 
Tabla 2. Cebadores y sondas usadas para la amplificación y diferenciación de CeMV. Adaptado de Grant et al. (2009) y RubioGuerri et al. (2013b). UPL, Universal Probe Library; FAM, 6-carboxyfluorescein Roche®.

\begin{tabular}{|c|c|c|c|}
\hline Virus & Cebador/sonda & Secuencia $5^{\prime}-3$ & Tamaño amplicón \\
\hline DMV y PMV & $\begin{array}{l}\text { DMV-UPL25F } \\
\text { DMV-UPL25-R } \\
\text { UPL\#25 }\end{array}$ & $\begin{array}{l}\text { TCTAGAGAATGTCAGGAGAAGTTCC } \\
\text { AAGCTCCTGCACATACAAGGAT } \\
\text { 6FAM-TGGAGGAG-dark quencher dye }\end{array}$ & 71 \\
\hline PWMV & $\begin{array}{l}\text { PWMV-UPL92-F } \\
\text { PWMV-UPL92-R } \\
\text { UPL\#92 }\end{array}$ & $\begin{array}{l}\text { GGAGGGGCAATGTATATAGGG } \\
\text { CAGCAGTATATCAACACGCATAG } \\
\text { 6FAM-CAGGAGCC-dark quencher dye }\end{array}$ & 74 \\
\hline DMV & $\begin{array}{l}\text { DMV-N-FP } \\
\text { DMV-N-RP } \\
\text { DMV-N-probe }\end{array}$ & $\begin{array}{l}\text { TGCCAGTACTCCAGGGAACATCCTTC } \\
\text { TTGGGTCGTCAGTGTTGTCGGACCGTT } \\
\text { Cy3-A+CA+CCAAA+AGGGA+CA-IBFQ }\end{array}$ & 173 \\
\hline PMV & $\begin{array}{l}\text { PMV-N-FP } \\
\text { PMV-N-RP } \\
\text { PMV-N-probe }\end{array}$ & $\begin{array}{l}\text { GTCTAGTGCTCCGGGGAATATCCCTA } \\
\text { CTGGATCATCAGCGTTGTCAGATTGCC } \\
\text { FAM-C+CA+TA+CCA+AG+AGGT-IBFQ }\end{array}$ & 173 \\
\hline DMV y PMV & $\begin{array}{l}\text { RT-FP (convencional) } \\
\text { C-term-RP (convencional) }\end{array}$ & $\begin{array}{l}\text { GGTCGITCTTACTTIGACCC } \\
\text { TTGGTT'TTCIAITAGCTTGGC }\end{array}$ & 461 \\
\hline
\end{tabular}

Las pruebas de ELISA tienen entre sus ventajas el hecho que adicional a no requerir manipulación de virus infecciosos, permiten la detección de anticuerpos específicos para Morbillivirus en sueros hemolizados que pueden ser citotóxicos y, podrían impedir la detección de anticuerpos a bajas diluciones en pruebas de seroneutralización (Van Bressem et al. 1998a,b). Sin embargo, estudios recientes concluyen que las pruebas de ELISA tienen una especificidad relativa, dado que el DMV y otros virus del género Morbillivirus, como el CDV de caninos y el PMV, tienen una relación genética y antigénica muy estrecha y por lo tanto reaccionan de forma cruzada (Tabla 3).

Todos los métodos anteriormente descritos son de uso aceptable dependiendo de las situaciones particulares para las cuales se necesite confirmar el diagnóstico, el momento del brote y los recursos

Tabla 3. Desempeño diagnóstico (sensibilidad y especificidad) de la prueba de ELISA competitivo en relación con la seroneutralización (VNT). Adaptado de Saliki et al. (2002).

\begin{tabular}{|c|c|c|c|c|}
\hline $\begin{array}{l}\text { Anticuerpos } \\
\text { específicos por VNT }\end{array}$ & Grupo animal & Sensibilidad & Especificidad & Índice Kappa \\
\hline \multirow{3}{*}{$\begin{array}{l}\text { CDV, PDV Y CDV/ } \\
\text { PDV }\end{array}$} & Canidos & 100.0 & 91.4 & 0.94 \\
\hline & Pinnipedos & 90.0 & 99.0 & 0.78 \\
\hline & Cánido/Pinnipedo & 98.8 & 98.4 & 0.95 \\
\hline $\begin{array}{l}\text { DMV, PMV, DMV/ } \\
\text { PMV }\end{array}$ & Cetáceos & 69.2 & 95.2 & 0.62 \\
\hline
\end{tabular}


disponibles. Sin embargo, a la hora de confirmar el diagnóstico del DMV, se sugiere la prueba de RT-PCR en tiempo real (Grant et al. 2009, Rubio-Guerri et al. 2013b), dada su mayor sensibilidad y especificidad que una RT-PCR convencional, además de su capacidad de diferenciación entre los diferentes CeMV.

Hasta ahora, no se tiene un tratamiento descrito que sea efectivo frente a la infección por el DMV. Los individuos afectados, que se encuentran varados son tratados con terapia de mantenimiento, pero la gran mayoría de estos animales terminan con desenlaces fatales (Stone et al. 2011). Esto aumenta la necesidad de implementar medidas de prevención y control para minimizar los efectos adversos en las poblaciones de cetáceos silvestres y en cautiverio; precisamente, una de las grandes preocupaciones y a la vez objetivos dentro de la medicina preventiva es la inmunización efectiva de las diferentes especies contra los patógenos; pensando en esto, se han utilizado ilegalmente diferentes vacunas contra el moquillo canino (a virus vivo modificado, virus inactivado o de subunidades) para tratar de contener los brotes e inmunizar a los animales. Sin embargo, a la fecha no existe ninguna vacuna con licencia para ser usada en cetáceos y pinnípedos.

En un esfuerzo por proteger dichas especies, el programa de conservación de mamíferos marinos de la fuerza naval de los Estados Unidos en cooperación con la industria y la academia, desarrollaron una vacuna de DNA que tiene como objetivo principal la protección contra el DMV mediante la efectiva inducción de respuesta tanto humoral como celular en delfines. El diseño de la vacuna se direccionó hacia dos genes blanco del DMV: la proteína de fusión y la hemaglutinina (Vaughan et al. 2007), dado que ya se ha demostrado el importante rol de dichas proteínas en la patogenicidad de los virus que comprenden la familia Paramyxoviridae.

En el mencionado estudio, dos grupos de delfines mulares, machos y hembras fueron inmunizados con los plásmidos vacunales (Pvr-DMV-F y Pvr- DMV-H) y con el vector solo como control negativo, usando un esquema de vacunación a la semana 0 , seguido por dos re-vacunaciones en la semanas 8 y 14 por vía intramuscular. Tal como se esperaba, los delfines tuvieron un aumento significativo en los títulos de anticuerpos específicos contra DMV, entre la semana 0 y la semana 20 (Vaughan et al. 2007). Aunque dichos resultados son un avance significativo para la implementación del proceso preventivo frente al DMV, se necesitan más investigaciones en el campo de la inmunología cetácea que permitan llevar estos resultados a la práctica en grandes poblaciones, en las cuales se garantice mediante seguimiento, una inmunización efectiva. Estas nuevas investigaciones, por ejemplo, podrían ampliar el conocimiento sobre los factores que contribuyen a la variación de la respuesta inmune, como la edad y la heterogeneidad genética; ya que por el momento sólo un estudio ha reportado cambios entre la edad y la función inmunitaria en delfines mulares (Blanchard et al. 2003). Toda esta información es de vital importancia a la hora de diseñar modelos de prevención y control para virus con alto potencial patogénico como el DMV.

\section{Conclusión}

Respecto a la importancia del morbilivirus de los Delfines (DMV) como patógeno re-emergente en la población mundial de delfines, es posible aseverar que no es sólo a la población de delfines a la que el DMV viene afectando, sino a otras especies, algunas incluso en peligro de extinción como la foca Monje del mediterráneo (Monachus monachus). Es de resaltar que durante la revisión de la literatura, se encuentran cada vez mayor cantidad de reportes sobre brotes y varamientos ocasionados por DMV tanto en América como en Europa. Por otro lado, la presente revisión nos permitió concluir que las poblaciones más afectadas a través de los años por este virus, han sido los delfines mulares y delfines listados de las costas de los EEUU y del mediterráneo. Actualmente, estas poblaciones se encuentran más vulnerables, debido a factores antropogénicos, menor disponibilidad de alimento y contaminación ambiental con sustancias tóxicas. Es de vital importancia, que los estudios de diagnóstico y caracterización tanto del DMV como de los demás CeMV, trasciendan del estudio de varamientos y conteo de tasas de ataque y de mortalidad de los diferentes brotes y sirvan para lograr conocer el panorama real de circulación de estos agentes en poblaciones silvestres de cetáceos, su epidemiología, los factores de riesgo y determinantes de emergencia y re-emergencia. Con toda esta información se podría desarrollar, lo más pronto posible, medidas efectivas de prevención y control que permitan mantener estas poblaciones en equilibrio biológico y que eviten la pérdida de vidas animales en especies tan importantes para los ecosistemas marinos. 


\section{Agradecimientos}

Nuestros agradecimientos al grupo de Investigación en Ciencias Veterinarias CENTAURO y al profesor Jairo Rivera del ARC Centre of Excellence for Coral Reefs Studies James Cook University/Australian Institute of Marine Science por sus correcciones y aportes al manuscrito. Este trabajo fue patrocinado por el "Programa de Sostenibilidad 2013-2014" del Comité para el Desarrollo de la Investigación - CODI de la Universidad de Antioquia.

\section{Conflictos de intereses}

Los autores declaran que no tiene ningún conflicto de intereses.

\section{Referencias}

Aguilar A, Raga JA (1993) The striped dolphin epizootic in the Mediterranean Sea. Ambio. 524-528

Barrett T, Visser IK, Mamaev L, Goatley L, Van Bressem MF, Osterhaust AD (1993) Dolphin and porpoise morbillivirus es are genetically distinct from. phocine distemper virus. Virology 193(2):1010-1012

Bellière E, Esperón F, Sánchez-Vizcaíno JM (2009) Estudio molecular de una nueva cepa de morbillivirus de cetáceo aislada de un calderon tropical. Revista Complutense de Ciencias Veterinarias 3(2):112-118

Bellière E, Esperón F, Sánchez-Vizcaíno JM (2011) Genetic comparison among dolphin morbillivirus in the 19901992 and 2006-2008 Mediterranean outbreaks. Infection, Genetics and Evolution 11(8):1913-1920 doi:10.1016/j. meegid.2011.08.018

Blanchard MT, Funke C, Stott JS (2003) Lymphocyte profile of free-ranging dolphins (Tursiops truncatus) in Sarasota Bay, Florida. In: Proceedings of the International Association for Aquatic Animal Medicine (IAAAM), Waikoloa, Hawaii

Daniel C (1995) The striped dolphin Stenella coeruleoalba epizootic in Greece, 1991-1992 Biological Conservation 74(2):143-145

Di Guardo G (2012) Morbillivirus-host interaction: lessons from aquatic mammals. Frontiers in microbiology 3:431 doi:10.3389/fmicb.2012.00431

Di Guardo G, Di Francesco CE, Eleni C, Cocumelli C, Scholl F, et al. (2013) Morbillivirus infection in cetaceans stranded along the Italian coastline: pathological, immunohistochemical and biomolecular findings. Research in Veterinary Science 94(1):132-7 doi:10.1016/j. rvsc.2012.07.030

Dierauf FL, Gulland F (2001) Viral diseases. In: Handbook of Marine Mammal Medicine. Second edition. Editorial CRC Press, New York, USA, pp 296-298
Domingo M, Ferrer L, Pumarola M, Marco A, Plana J, et al. (1990) Morbillivirus in dolphins. Nature 348:21

Domingo M, Vilafranca M, Visa J, Prats N, Trudgett A, Visser I (1995) Evidence for chronic morbillivirus infection in the Mediterranean striped dolphin (Stenella coeruleoalba). Veterinary Microbiology 44:229-239

Domingo M, Visa J, Pumarola M, Marco AJ, Ferrer L, et al. (1992) Pathologic and immunocytochemical studies of morbillivirus infection in striped dolphins (Stenella coeruleoalba).Veterinary Pathology 29(1):1-10

Duignan PJ, House C, Odell DK, Wells RS, Hansen W, et al. (1996) Morbillivirus in bottlenose dolphins: evidence for recurrent epizootics in the western Atlantic and Gulf of Mexico. Marine Mammal Science 12:499-515

Fernández A, Esperón F, Herraéz P, de Los Monteros AE, Clavel C, et al. (2008) Morbillivirus and pilot whale deaths, Mediterranean Sea. Emerging Infectious Diseases. 14(5):792794 doi:10.3201/eid1405.070948

Forcada J, Aguilar A, Hammond PS, Pastor X, Aguilar R (1994) Distribution and numbers of striped dolphins in the western Mediterranean Sea after the 1990 epizootic outbreak. Marine Mammal Science 10:137-150

Fowler M, Cubas Z (2001) Orders Cetacea and Pinnipedia (whales, dolphins, porpoises, seals, fur seals, sea lions). In: Biology, Surgery and Medicine of South American Wild Animals. Saunders Company, pp 332-352

Geraci JR (1989) Clinical investigation of the 1987-1988 mass mortality of bottlenose dolphins along the US Central and south Atlantic coast. Final Report to the National Marine Fisheries Service, US Navy (Office of Naval Research) and Marine Mammal Commission, pp 63-64

Grant RJ, Banyard AC, Barrett T, Saliki JT, Romero CH (2009) Realtime RT-PCR assays for the rapid and differential detection of dolphin and porpoise morbillivirus es. Journal of virological methods 156(1):117-123 doi:10.1016/j. jviromet.2008.11.008

Gozalbes P, Jiménez J, Raga JA, Esteban JA, Tomás J, Gómez JA, Eymar J (2010) Cetáceos y tortugas marinas en la Comunidad Valenciana. 20 años de seguimiento. Collecció Treballs Tècnics de Biodiversitat, 3. Consellería de Medio Ambiente, Agua, Urbanismo y Vivienda. Generalitat Valenciana. Valenciana, España

Keck N, Kwiatek O, Dhermain F, Dupraz F, Boulet H, et al. (2010) Resurgence of Morbillivirus infection in Mediterranean dolphins off the French coast. The Veterinary record 166(21):654-655 doi:10.1136/vr.b4837

Kennedy S (1998) Morbillivirus infections in aquatic mammals. Journal of comparative pathology 119(3):201-225

Lahvis G, Wells RS, Kuehl DW, Stewart JL, Rhinehard HL, Via CS (1995) Decreased lymphocyte response in free-ranging bottlenose dolphins (Tursiops truncatus) are associated with increased concentrations of PCBs and DDT in peripheral blood. Environmental Health Perspectives 103(Suppl. 4):67-72 
Ludlow M, Rennick LJ, Nambulli S, de Swart RL, Paul Duprex W (2014) Using the ferret model to study morbillivirus entry, spread, transmission and crossspecies infection. Current Opinion in Virology 4:15-23 doi:10.1016/j.coviro.2013.11.001

McLellan WA, Friedlander AS, Mead JG, Potter CW, Pabst DA (2002) Analysing 25 years of bottlenose dolphin (Tursiops truncatus) strandings along the Atlantic coast of the USA: do historic records support the coastal migratory stock hypothesis? Journal of Cetacean Research and Management 4(3):297-304

Mazzariol S, Marcer F, Mignone W, Serracca L, Goria M, et al. (2012) Dolphin Morbillivirus and Toxoplasma gondii coinfection in a Mediterranean fin whale (Balaenoptera physalus). BMC veterinary research 8(1):20 doi:10.1186/1746-6148-8-20

Mazzariol S, Peletto S, Mondin A, Centelleghe C, Di Guardo G, et al. (2013) Dolphin morbillivirus infection in a captive harbor seal (Phoca vitulina). Journal of clinical microbiology 51(2):708-11 doi:10.1128/JCM.02710-12

Mori C, Morsey B, Levin M, Gorton TS, De Guise S (2008) Effects of organochlorines, individually and in mixtures, on B-cell proliferation in marine mammals and mice. Journal of Toxicology and Environmental Health 71(4):266-275 doi:10.1080/15287390701612860

Mori C, Morsey B, Levin M, Nambiar PR, De Guise S (2006) Immunomodulatory effects of in vitro exposure to organochlorines on T-cell proliferation in marine mammals and mice. Journal of Toxicology and Environmental Health 69:283-302

NOAA - National Marine Fisheries Service (2014) Bottlenose Dolphin Unusual Mortality Event in the Mid-Atlantic. http://www.nmfs.noaa.gov/pr/health/mmume/ midatldolphins2013.html Retrieved Abril 23, 2014

O'Mara FP, Coyle JE, Drennan MJ, Young P, Caffrey PJ, et al. (1999) Morbillivirus es in Mediterranean monk seals. Veterinary microbiology 69(1):19-21

Osterhaus AD, Visser IK, de Swart RL, Van Bressem MF, Van de Bildt, MW, et al. (1992) Morbillivirus threat to Mediterranean monk seals? The Veterinary record 130(7):141-142

Raga JA, Banyard A, Domingo M, Corteyn M, Van Bressem MF, et al. (2008) Dolphin morbillivirus epizootic resurgence, Mediterranean Sea. Emerging infectious diseases 14(3):471-473 doi:10.3201/eid1403.071230

Reidarson TH, McBain J, House C, King DP, Stott JL, et al. (1998) Morbillivirus infection in stranded common dolphins from the Pacific Ocean. Journal of wildlife diseases 34(4):771-776

Rima BK, Collin AM, Earle JA (2005) Completion of the sequence of a Cetacean morbillivirus and comparative analysis of the complete genome sequences of four morbillivirus es. Virus Genes 30(1):113-119
Rosel PE, Hansen L, Hohn AA (2009) Restricted dispersal in a continuously distributed marine species: common bottlenose dolphins Tursiops truncatus in coastal waters of the western North Atlantic. Molecular Ecology 18(24):50305045 doi:10.1111/j.1365-294X.2009.04413.x

Rubio-Guerri C, Melero M, Esperón F, Bellière EN, Arbelo M, et al. (2013a) Unusual striped dolphin mass mortality episode related to cetacean morbillivirus in the Spanish Mediterranean Sea. BMC veterinary research 9(1):106 doi:10.1186/1746-6148-9-106

Rubio-Guerri C, Melero M, Rivera-Arroyo B, Bellière EN, Crespo JL, et al. (2013b) Simultaneous diagnosis of Cetacean morbillivirus infection in dolphins stranded in the Spanish Mediterranean sea in 2011 using a novel Universal Probe Library (UPL) RT-PCR assay. Veterinary microbiology 165(1-2):109-114 doi:10.1016/j. vetmic.2012.12.031

Saliki JT, Cooper EJ, Gustavson JP (2002) Emerging Morbillivirus Infections of Marine Mammal. Annals of the New York Academy of Sciences 969(1):51-59

Scott GP, Burn DM, Hansen LJ (1998) The dolphin dieoff Long term effects and recovery of the population. Oceans '88 Proceedings 3:819-823

Seimon TA, Miquelle DG, Chang TY, Newton AL, Korotkova I, et al. (2013) Canine distemper virus: an emerging disease in wild endangered Amur tigers (Panthera tigris altaica). MBio 4(4):410-13 doi:10.1128/ mBio.00410-13

Sinclair AR, Fryxell JM, Caughley G (2006) Parasites and pathogens. In Wildlife ecology, conservation, and management. Oxford: Wiley-Blackwell, pp 179-195

Soto S, Alba A, Ganges L, Vidal E, Raga JA, et al. (2011) Post-epizootic chronic dolphin morbillivirus infection in Mediterranean striped dolphins Stenella coeruleoalba. Diseases of aquatic organisms 96(3):187-94 doi:10.3354/ dao02387

Stone BM, Blyde DJ, Saliki JT, Blas-Machado U, Bingham J, et al. (2011) Fatal cetacean morbillivirus infection in an Australian offshore bottlenose dolphin (Tursiops truncatus). Australian Veterinary Journal 89(11):452-7 doi:10.1111/j.1751-0813.2011.00849.x

Taubenberger JK, Tsai MM, Kraft AE, Lichy JH, Reid AH, Schulman FY (1996) Two morbillivirus implicates in bottlenose epizootics. Emerging Infectious Diseases 2(3):213

Taubenberger JK, Tsai MM, Atkin TJ, Fanning TG, Krafft AE, et al. (2000) Molecular genetic evidence of a novel morbillivirus in a long-finned pilot whale (Globicephalus melas). Emerging Infectious Diseases 6(1):42-45

Van Bressem MF, Van Waerebeek K, Fleming M, Barrett T (1998a) Serological evidence of morbillivirus infection in small cetaceans from the Southeast Pacific. Veterinary microbiology 59(2-3):89-98 
Van Bressem MF, Jepson P, Barrett T (1998b) Further insight on the epidemiology of cetacean morbillivirus in the Northeastern Atlantic. Marine Mammal Science 14(3):605613

Van Bressem MF, Raga JA, Di Guardo G, Jepson PD, Duignan P, et al. (2009) Emerging infectious diseases in cetaceans worldwide and the role of environmental stressors. Diseases of aquatic organisms 86(2):143-57 doi:10.3354/dao02101
Van de Bildt MW, Kuiken T, Osterhaus AD. (2005). Cetacean morbilliviruses are phylogenetically divergent. Archives of Virology 150(3):577-83. doi:10.1007/s00705-0040426-4

Vaughan K, Del Crew J, Hermanson G, Wloch MK, Riffenburgh RH, et al. (2007) A DNA vaccine against dolphin morbillivirus is immunogenic in bottlenose dolphins.Veterinary immunology and immunopathology 120 (3-4):260-266 doi:10.1016/j.vetimm.2007.06.036
Morbillivirus de los Delfines: Patógeno re-emergente en la población de cetáceos

Resumen. El morbillivirus de los Delfines (DMV) es uno de los agentes más patógenos de los cetáceos; descrito por primera vez a finales de los años 80 , y desde entonces ha venido causando brotes que afectan no sólo a las poblaciones de delfines sino también a las de otros cetáceos tanto en las costas de los EEUU como del mediterráneo. En la presente revisión se realizó una búsqueda de literatura científica en MedLine,

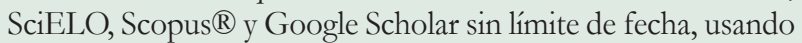
descriptores tales como Cetacean morbillivirus, Pilot Whale morbillivirus, Dolphin morbilivirus, strandings, con el objetivo de presentar una actualización crítica sobre los aspectos básicos, clínicos y epidemiológicos de una de las infecciones emergentes más importantes para la fauna cetácea mundial, haciendo especial énfasis en las alternativas actuales para su diagnóstico y control. Con base en la información recopilada se concluye que hace falta no solo estudiar y cuantificar los varamientos, sino desarrollar conciencia sobre la importancia de la infección en los cetáceos y poner en marcha de planes efectivos de control que permitan mantener estas poblaciones en equilibrio biológico.

Palabras clave: Epidemiología; morbillivirus de los cetáceos; Morbillivirus de la ballena piloto; Paramixoviridae; RT-PCR.
Morbillivirus dos Golfinhos: Patógeno re-emergentes em populaçóes de cetáceos

Resumo. O morbillivirus dos Golfinhos (DMV) é um dos mais importantes agentes patógenos dos cetáceos; foi descrita pela primeira vez no final dos anos 80 , e desde então tem provocado surtos que afetam tanto as populações de golfinhos quanto as de outros cetáceos nas duas regiões litorais dos EUA mesmo que no mediterrâneo. Nesta revisão, a pesquisa bibliográfica foi realizada no Medline, Scielo, Scopus e Google Scholar ${ }^{\circledR}$, usando descritores como Cetacean morbillivirus, Pilot Whale morbillivirus, Dolphin morbilivirus, strandings, objetivando apresentar uma atualização crítica e básica dos aspectos clínicos e epidemiológicos das infeções emergentes mais importantes para a vida dos cetáceos no mundo, fazendo especial ênfase nas atuais alternativas de diagnostico y controle. Concluísse, na base destas informações que além de estudar e quantificar os animais encalhados deve se desenvolver a consciência da importância da infecção em cetáceos e implementar planos de gestão eficazes que mantenham as populações em equilíbrio biológico.

Palavras-chave: Epidemiologia; morbilivírus do cetáceos; morbilivírus da baleia piloto; Paramyxoviridae; RT-PCR. 